\title{
Clinical parameters predictive of enlargement of melanocytic choroidal lesions*
}

\author{
JAMES J AUGSBURGER,' ROBERT P SCHROEDER,' CARLA TERRITO,' \\ JOHN W GAMEL, ' AND JERRY A SHIELDS'
}

From the 'Oncology Service, Wills Eye Hospital, Department of Ophthalmology, Jefferson Medical College, Thomas Jefferson University, Philadelphia, Pennsylvania, USA, and the 'Department of Ophthalmology, University of Louisville School of Medicine, Louisville, Kentucky, USA

SUMmary The authors followed up 197 melanotic choroidal lesions (62 categorised as benign naevi, 76 classified as suspicious naevi, 41 diagnosed as dormant melanomas, and 18 categorised as active melanomas) left untreated after their initial clinical documentation. Thirty-nine of these lesions enlarged during a five-year follow-up interval (cumulative proportion of lesions that enlarged $=26 \cdot 2 \%$ by Kaplan-Meier method). Individual clinical parameters predictive of lesion enlargement $(p<0.01)$ included larger size of the lesion, especially lesion thickness, presence of retinal detachment, location of the lesion's posterior margin within 2 disc diameters of the optic disc, presence of symptoms, and presence of orange pigment clumps on the lesion's surface. The best combination of these parameters for prediction of lesion enlargement, as identified by multivariate Cox regression analysis, consisted of thickness of the lesion, retinal detachment, and symptoms. The five-year incidence of lesion enlargement for patients with none of these prognostic parameters was $5 \cdot 8 \%$, while that for patients with all three unfavourable parameters simultaneously was $90 \cdot 6 \%$.

In spite of improvements in ophthalmic diagnostic methods, clinical differentiation between benign choroidal naevi and malignant melanomas is not completely reliable. ': Clinical parameters currently used to assist in this distinction include lesion size, symptoms, presence or absence of orange pigment and drusen on the surface of the lesion, extent of serous retinal detachment, and lesion enlargement. ${ }^{1-4}$ Of these parameters, enlargement of the lesion is generally regarded as the most reliable predictor of malignant potential in a melanocytic choroidal lesion. ${ }^{6 \mathrm{x}}$

Growth of a choroidal lesion initially diagnosed as a benign naevus usually prompts reclassification of the lesion as a melanoma and consideration of

\footnotetext{
* Presented in part at the eleventh annual scientific meeting of the Macula Society, Tuscon. Arizona, 26 February 1988, and at the annual spring meeting of the Association for Research in Vision and Ophthalmology, Sarasota, Florida, 5 May 1988.
}

Correspondence to either Jerry A Shields, MD. Director, Oncology Service. Wills Eye Hospital, or to James J Augsburger, MD, Retina Service, 9th and Walnut Streets, Philadelphia, PA 19107. USA. treatment. ${ }^{16}$ However, a number of studies have shown that only a small proportion of clinically diagnosed choroidal naevi and small melanomas enlarge during short-term follow up. ${ }^{6 \times 4}$ Surprisingly, little is known about which clinical parameters predict lesion enlargement. To study this question the authors investigated the ability of various clinical parameters assessed at initial diagnostic examination to predict enlargement of melanocytic choroidal lesions.

\section{Patients and methods}

The authors surveyed the clinical records of all new patients examined at the Oncology Service of Wills Eye Hospital between 1 July 1980 and 30 June 1982, with the aid of a computerised patient database. The records of all patients diagnosed on initial examination as having a choroidal lesion classified as a freckle, non-suspicious naevus, suspicious naevus, dormant melanoma, or active melanoma (as defined by Shields"') were reviewed. Those records indicating that the patient had been managed, at least initially, 
by observation without treatment were selected for data abstraction.

Clinical parameters extracted from the written clinical records of these patients included age at presentation, sex and race, presence or absence of symptoms attributable to the tumour, duration of any symptoms attributable to the lesion, and best corrected distance visual acuity (Snellen notation). Chart notations, fundus diagrams, and fundus photographs were used to assess colour of the lesion, lesion basal diameters (estimated to the nearest $0.5 \mathrm{~mm}$ ) and thickness (measured by standardised A-scan ultrasonography), location of the anterior margin of the lesion in relation to the equator, location of the posterior margin of the lesion relative to the optic disc and foveola, and presence and extent or absence of serous non-rhegmatogenous retinal detachment. The presence or absence of orange pigment clumps, drusen, and black retinal pigment epithelial pigment clumps on the surface of the lesion were assessed on the fundus photographs of each lesion relative to a set of standard fundus photographs (Figs. 1-3). If the extent of any of these features was as great as or greater than the extent of the features depicted in the standard photographs, the feature was coded present.

The result of interest in this study was recorded enlargement of the lesion verified by fundus photography, ultrasonography, or both. For each patient whose melanocytic choroidal lesion enlarged during follow-up the duration of follow-up was computed as the interval between the patient's initial diagnostic examination and the date when enlargement of the lesion was recorded. In contrast, for patients whose lesion did not enlarge during observation the duration of follow-up was computed as the interval between the initial examination and the patient's most recent follow-up visit.

Conventional descriptive statistics were used to summarise the recorded parameters in these patients. The cumulative proportion of the melanocytic choroidal lesions which enlarged during follow-up was computed by the method of Kaplan and Meier. ${ }^{.1}$ Patients with lesions that enlarged were compared by unpaired $t$ testing and $\chi^{2}$ analysis with patients with lesions that did not.

Cox proportional hazards modelling ${ }^{12}$ was used to assess the prognostic value of the recorded clinical parameters for prediction of subsequent lesion enlargement. Both univariate and multivariate regressions were explored. For the multivariate analysis a special algorithm written by one of us (JWG) was used to test all possible two-term and three-term Cox regressions. A multivariate regression was considered to be significant only if all component variables entered at a statistically signi- ficant level. Because of the exploratory nature of this study and the multiplicity of assessd variables the authors specified a conservative significance level of $\alpha=0.01$ in advance of data analysis. The best twoterm and three-term models were determined from among the significant Cox models by the following method: (a) a prognostic index (hazard ratio) was computed for each case from the $\beta$ coefficients estimated for the particular Cox model; $(b)$ the cases were divided into low-risk and high-risk groups according to the mean hazard ratio predicted from the $\beta$ coefficients computed from the Cox model; and (c) five-year Kaplan-Meier lesion non-enlargement rates for the two groups were computed for each of the significant models.

\section{Results}

The authors identified 197 lesions which fulfilled the inclusion criteria of this study. Our initial clinical diagnosis was freckle or benign naevus in $62(31.5 \%)$, suspicious naevus in $76(38.6 \%)$, dormant melanoma in $41(20 \cdot 8 \%)$, and active melanoma in $18(9 \cdot 1 \%)$. The affected patients ranged in age from 15 to 87 years (mean $58 \cdot 1$, SD 13.4 years). All of the patients were white. Seventy-nine of the lesions $(40 \cdot 1 \%)$ occurred in males and $118(59.9 \%)$ in females. One hundred and one of the 197 lesions $(51 \cdot 3 \%)$ involved the right eye and 96 involved the left $(48.7 \%)$. One hundred and forty-six of the 197 choroidal lesions were asymptomatic, but 51 of the lesions $(25.9 \%)$ were associated with visual symptoms.

The lesions of interest ranged in size from 0.5 to $15.5 \mathrm{~mm}$ in basal diameter (mean 5.2 , SD $3.1 \mathrm{~mm}$ ) and from flat to $8.0 \mathrm{~mm}$ in thickness (mean 1.3 , SD $1.3 \mathrm{~mm}$ ). Pigmentation of the lesion was categorised as amelanotic in nine $(4.6 \%)$, intermediate in 64 $(32.5 \%)$, and darkly melanotic in $124(62 \cdot 9 \%)$. One hundred and forty-eight of the 179 lesions $(75 \cdot 1 \%)$ were located entirely posterior to the equator, while $49(24.9 \%)$ either straddled the equator or were entirely pre-equatorial. The posterior margin of the lesion was over 2 disc diameters from the optic disc in 128 eyes $(65.0 \%)$, within 2 disc diameters from the optic disc in 48 eyes $(24.4 \%)$, and touching the optic disc margin in 21 eyes $(10 \cdot 6 \%)$. Nineteen of the 197 lesions $(9.6 \%)$ involved the subfoveolar choroid; 44 additional lesions $(22.3 \%)$ were located within 2 disc diameters of the foveola, and 134 lesions $(68.0 \%)$ were more than 2 disc diameters from the foveola.

One hundred and sixty-one of the 197 choroidal lesions had no associated retinal detachment. Of the remaining lesions $26(13 \cdot 3 \%)$ were associated with a localised collection of subretinal fluid extending no more than 1 disc diameter beyond any margin of the 


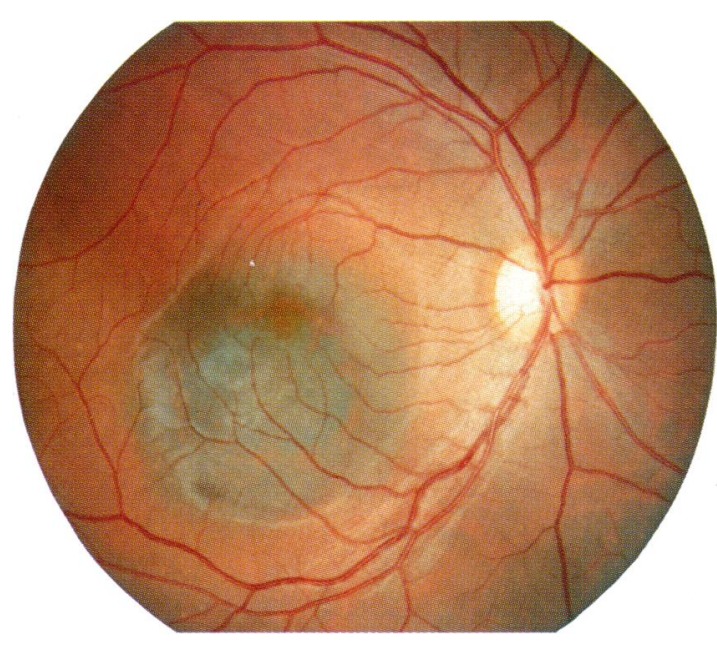

Fig. 1 Standard photograph used to identify orange pigment clumps on the surface of a melanocytic choroidal lesion.

lesion, and nine $(4.6 \%)$ were associated with a more extensive non-rhegmatogenous retinal detachment.

Orange pigment clumps equal to or greater in extent than those depicted in our standard photograph (Fig. 1) were identified on the surface of 17 of the 197 choroidal lesions $(8 \cdot 6 \%)$. In contrast, drusen equal to or greater in extent than those shown in our standard photograph (Fig. 2) were present on 83 $(42 \cdot 1 \%)$ of the 197 lesions, and black retinal pigment epithelial pigment clumps equal to or greater in extent than those shown in our standard photograph (Fig. 3) were found on only 25 of the 197 lesions $(12 \cdot 7 \%)$.

Thirty-nine of the 197 lesions enlarged during the

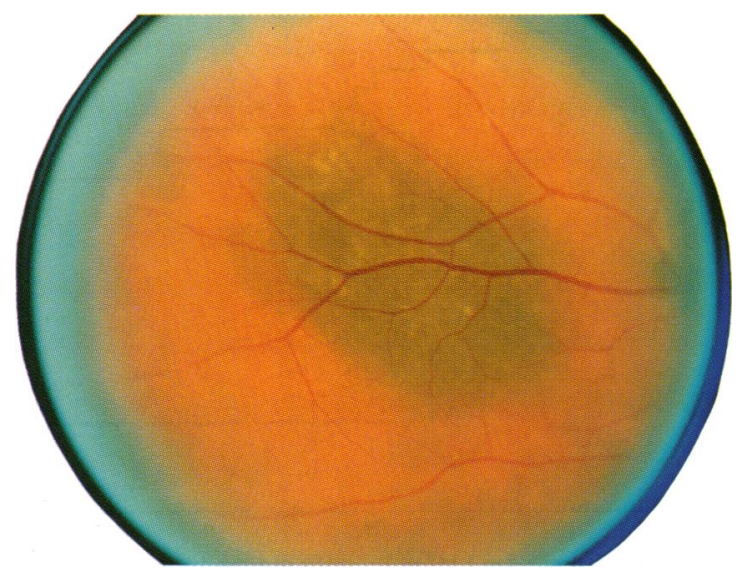

Fig. 2 Standard photograph used to identify drusen on the surface of a melanocytic choroidal lesion.

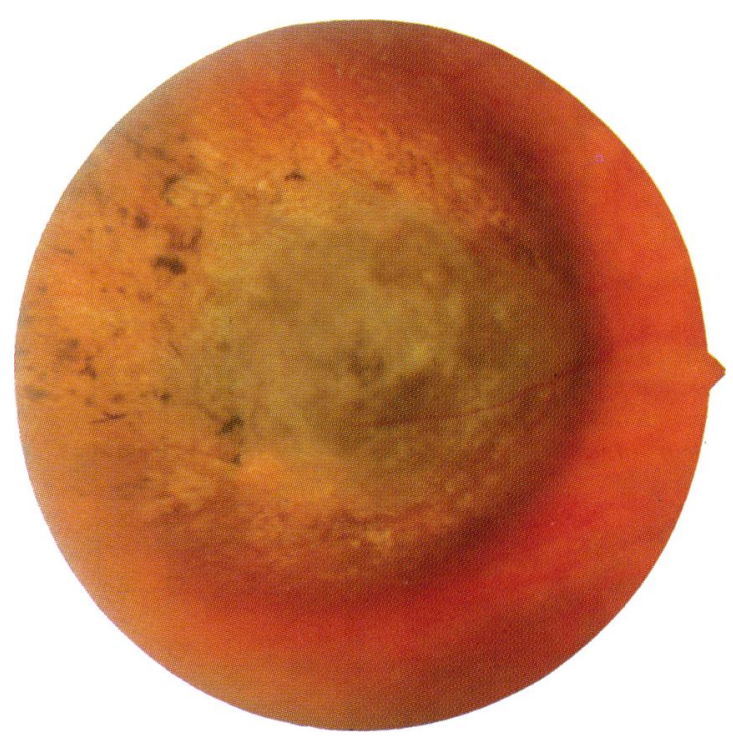

Fig. 3 Standard photograph used to identify retinal pigment epithelial pigment clumping on the surface of a melanocytic choroidal lesion.

interval of study. The Kaplan-Meier estimate of the cumulative proportion of lesions enlarging within five years was $26 \cdot 2$ (SE $4 \cdot 8$ )\% (Fig. 4). The proportion of lesions which did not enlarge as a function of our initial clinical diagnosis is presented graphically as Fig. 5. This figure indicates that only $4.5 \%$ of melanocytic choroidal lesions diagnosed clinically as 'benign naevi' enlarged during five years of followup. In contrast, $14.2 \%$ of lesions diagnosed as 'suspicious naevi', $50 \cdot 1 \%$ of lesions categorised as 'dormant melanomas', and $86 \cdot 1 \%$ of lesions classified as 'active melanomas' enlarged during the same period of follow up.

Table 1 compares the 39 lesions which enlarged with the 158 which remained unchanged in size with regard to the distribution of the assessed clinical variables. The lesions that grew were larger and located nearer the optic disc than those that remained stable. Furthermore, a higher proportion of lesions that enlarged had associated subretinal fluid, prominent orange pigment clumps on their surface, and related symptoms than did lesions that remained unchanged in size.

Table 2 presents the results of univariate prognostic factor analysis by Cox proportional hazards modelling. Larger size of the lesion (especially lesion thickness), more extensive retinal detachment, more posterior location of the lesion's posterior margin, presence of symptoms, and orange pigment clumps on the lesion's surface were all significant individual predictors $(p<0 \cdot 01)$ of lesion enlargement. 
Fig. 4 Kaplan-Meier estimate of the cumulative proportion of 197 melanocytic choroidal lesions which did not enlarge during five years of follow-up.

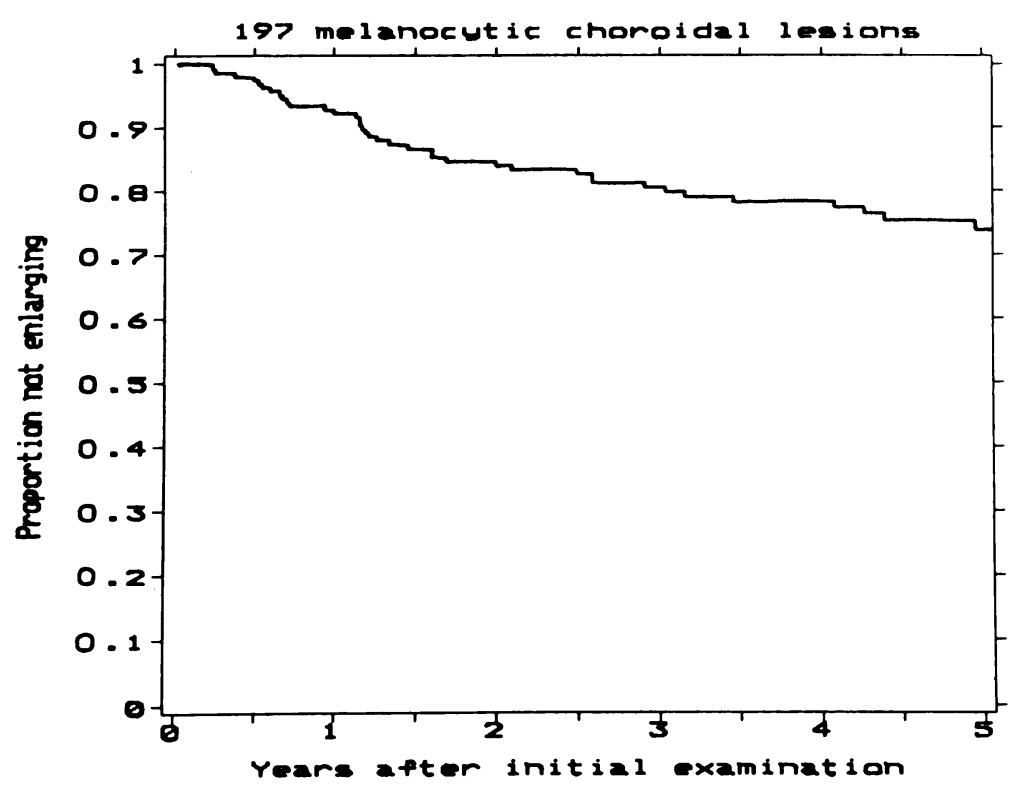

The best multivariate statistical model for predicting lesion enlargement was a three-term regression incorporating thickness of the lesion, extent of retinal detachment, and symptoms (logrank test $t=$ $7 \cdot 5, \mathrm{p}<0.0001)$. This model allowed us to divide the 197 cases into a low-risk group of 160 lesions with a five-year lesion enlargement rate of $10.9 \%$ and a high-risk group of 37 lesions with a five-year lesion enlargement rate of $86 \cdot 2 \%$. The best alternative multivariate model not incorporating lesion thickness was a three-term regression incorporating the lesion's basal diameter, symptoms, and age of the patient. The best alternative regression not incorporating any lesion size parameter was a two-term regression including symptoms and orange pigment on the lesion's surface.
Fig. 5 Relationship between time to lesion enlargement and initial clinical diagnosis of 197 melanocytic choroidal lesions. Lesions were categorised as freckles and non-suspicious naevi (NN), suspicious naevi $(S N)$, dormant melanomas (DM), or active melanomas ( $A M)$.

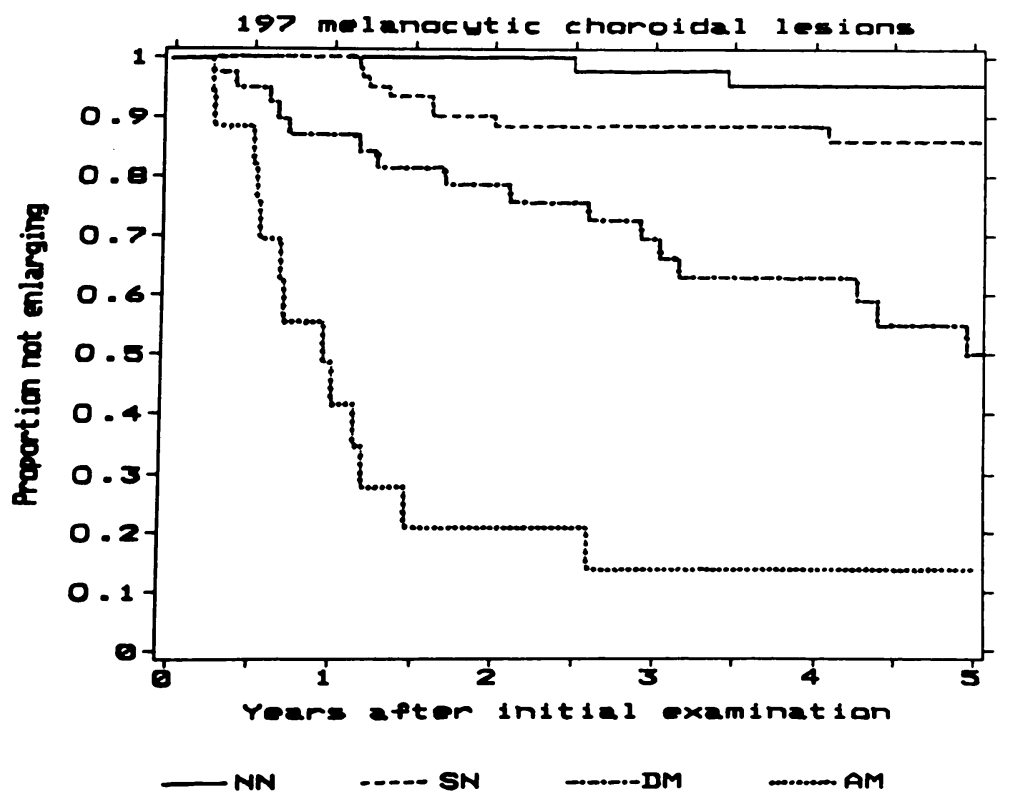


Table 1 Distribution of clinical variables by lesion enlargement category for 197 patients with a melanocytic choroidal lesion

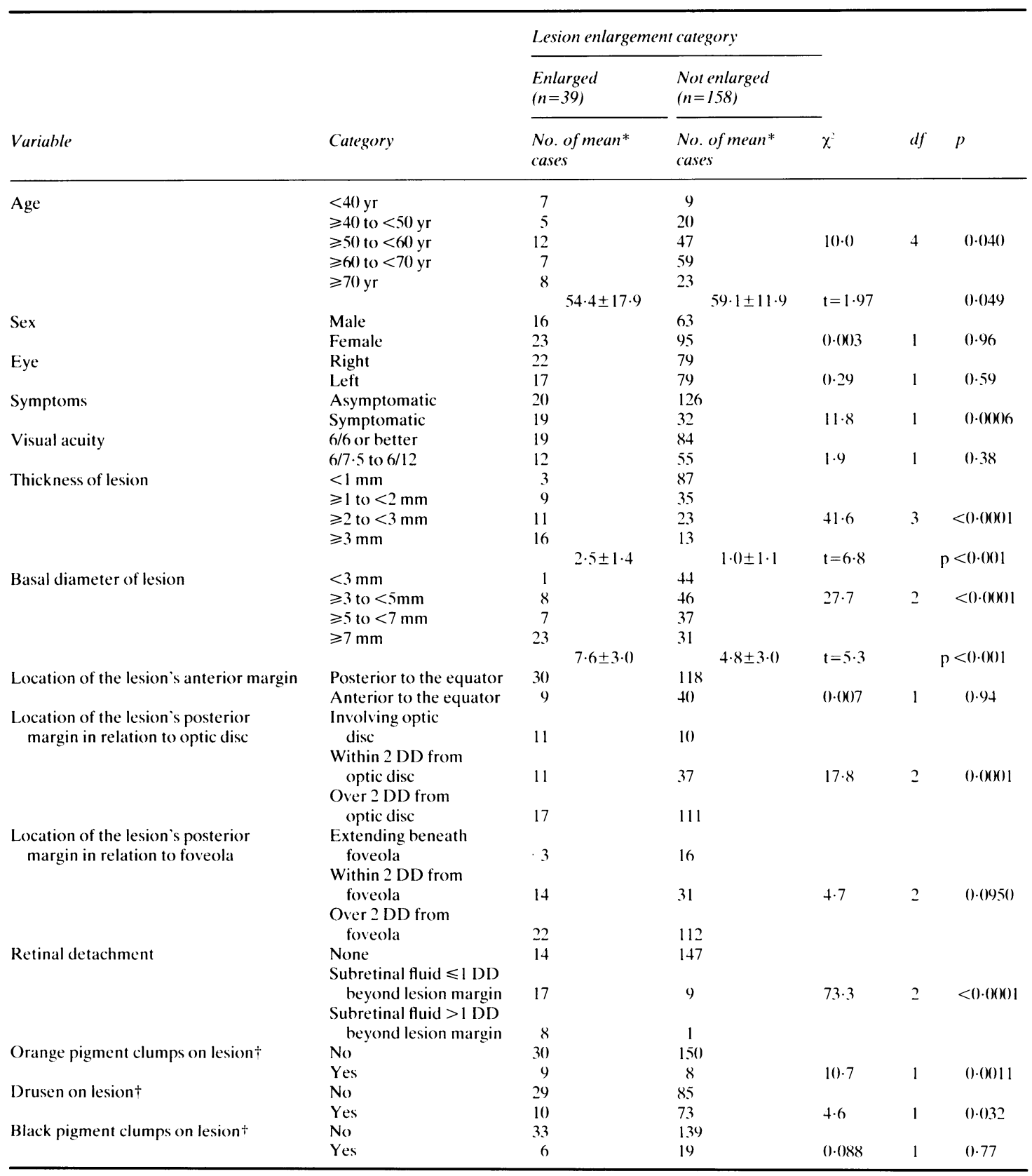

* Mean values of variable are shown only for normally distributed variables recorded on a standard interval scale.

$\dagger$ Variable assessed with reference to standard photograph.

$\mathrm{DD}=$ disc diameter, approximately $1.75 \mathrm{~mm}$.

To simplify the presentation of our prognostic factor results we compiled a table (Table 3 ) showing the incidence of lesion enlargement through 2.5 and 5 years as a function of the presence or absence of the three parameters identified in our best Cox model (see preceding paragraph). This table is a con- 
Table 2 Prognostic factor assessment by univariate Cox modelling

\begin{tabular}{|c|c|c|c|c|}
\hline variable & $\begin{array}{l}\text { beta } \\
\text { coefficient }\end{array}$ & $\begin{array}{l}\text { standard } \\
\text { error of } \beta\end{array}$ & $\mathbf{t}$ & $p$ \\
\hline Retinal detachment & $1 \cdot 69$ & $0 \cdot 20$ & $8 \cdot 3$ & $<0 \cdot 0001$ \\
\hline Thickness of lesion & 0.64 & $0 \cdot 10$ & $6 \cdot 7$ & $<0 \cdot(0001$ \\
\hline Basal diameter of lesion & $0 \cdot 21$ & $0 \cdot(04$ & 4.9 & $<0 \cdot(0001$ \\
\hline \multicolumn{5}{|l|}{$\begin{array}{l}\text { Location of lesion's } \\
\text { posterior margin }\end{array}$} \\
\hline relative to optic disc & -0.74 & $0 \cdot 19$ & $3 \cdot 8$ & $0 \cdot 0001$ \\
\hline Symptoms & $1 \cdot() 8$ & $0 \cdot 32$ & $3 \cdot 4$ & $0 \cdot 0007$ \\
\hline \multicolumn{5}{|l|}{ Orange pigment clumps } \\
\hline on lesion & $1 \cdot 23$ & $0 \cdot 38$ & $3 \cdot 2$ & $0 \cdot(0) 12$ \\
\hline Drusen on lesion & -0.84 & $0 \cdot 37$ & $2 \cdot 3$ & $0 \cdot() 22$ \\
\hline Age of patient & $-0 \cdot() 2$ & $0 \cdot 01$ & 1.9 & $0 \cdot 0.52$ \\
\hline Colour of lesion & -0.42 & $0 \cdot 25$ & $1 \cdot 7$ & $0 \cdot(991$ \\
\hline Visual acuity & $-0 \cdot 08$ & 0.05 & $1 \cdot 6$ & $0 \cdot 11$ \\
\hline \multicolumn{5}{|l|}{ Location of lesion's } \\
\hline relative to foveola & $-0 \cdot 23$ & $0 \cdot 22$ & $1 \cdot 1$ & 0.29 \\
\hline \multicolumn{5}{|l|}{ Black pigment clumps } \\
\hline on lesion & $0 \cdot 30$ & 0.44 & 0.67 & 0.51 \\
\hline Eye & $-0 \cdot 19$ & $0 \cdot 32$ & 0.58 & 0.56 \\
\hline Sex & $(0 \cdot 11$ & $0 \cdot 33$ & 0.35 & 0.73 \\
\hline $\begin{array}{l}\text { Location of lesion's } \\
\text { anterior margin }\end{array}$ & $-0 \cdot(07$ & 0.38 & 0.18 & 0.86 \\
\hline
\end{tabular}

venient way of defining which melanocytic choroidal lesions are high risk for enlargement. From this table one can see that the five-year incidence of lesion enlargement for patients with none of these prognostic parameters was $5 \cdot 8 \%$, while that for patients with all three unfavourable parameters simultaneously was $90 \cdot 6 \%$.

\section{Discussion}

This study shows by survival analysis that the majority of melanocytic choroidal lesions classified as naevi do not enlarge appreciably during five years of follow-up. In contrast, the majority of lesions classified clinically as melanomas do enlarge during a five year follow-up period. Neither of these findings is surprising.

Most of the results of our analysis by univariate prognostic factor agree with Gass's findings. ${ }^{.}$Lesion thickness appeared to be a better indicator of growth potential than was lesion basal diameter in both Gass's series and in ours. Similarly, the presence of drusen appeared to be predictive of lesion dormancy in both series. Symptoms attributable to the lesion, prominent orange pigment clumps on the lesion's surface, and retinal detachment were also predictive of lesion enlargement in both series.

The predictive value of location of the lesion's posterior margin shown in this study was unexpected. The significance of location of the lesion's posterior margin in both univariate and multivariate models, however, suggests that this parameter may convey useful prognostic information about lesion enlargement. On the other hand this parameter may be significant only because it is more difficult for ophthalmologists to identify subtle enlargement of peripheral lesions than of posterior ones. Assessment of the prognostic value of this parameter in other data sets may clarify its true significance.

Although the best multivariate model for prediction of lesion enlargement in this group of patients was the three term regression presented in the 'Results' section, several other three-term regressions were of almost equivalent predictive value. It is certainly possible that an alternative model may prove to be a more appropriate and reliable regression for prediction of lesion enlargement in other studies of melanocytic choroidal lesions. The three parameters included in our model are not necessarily the most important ones in all patients with melano-

Table 3 Cumulative actuarial proportion of 197 melanocytic choroidal lesions which enlarged during follow-up as a function of the presence of identified prognostic factors

\begin{tabular}{|c|c|c|c|c|c|c|c|}
\hline \multicolumn{8}{|c|}{ Prognostic Factors } \\
\hline \multirow[t]{2}{*}{ Symptoms } & \multirow[t]{2}{*}{$\begin{array}{l}\text { Thickness of lesion } \\
>1.5 \mathrm{~mm}\end{array}$} & \multirow[t]{2}{*}{ Retinal detachment } & \multirow[t]{2}{*}{$\begin{array}{l}\text { Number of prognostic } \\
\text { factors present }\end{array}$} & \multicolumn{2}{|c|}{$\begin{array}{l}2 \cdot 5 \text {-year incidence of } \\
\text { lesion enlargement } \\
\text { (percentage) }\end{array}$} & \multicolumn{2}{|c|}{$\begin{array}{l}\text { 5-year incidence of } \\
\text { lesion enlargement } \\
\text { (percentage) }\end{array}$} \\
\hline & & & & rate & standard error & rate & standard error \\
\hline \multirow{4}{*}{ Yes } & & & 0 & $4 \cdot 1$ & $2 \cdot 3$ & $5 \cdot 8$ & $2 \cdot 8$ \\
\hline & & & 1 & $38 \cdot 0$ & $7 \cdot 1$ & 4()$\cdot 7$ & $7 \cdot 3$ \\
\hline & Yes & & 1 & $35 \cdot 8$ & $6 \cdot 5$ & $54 \cdot 1$ & $7 \cdot 7$ \\
\hline & & Yes & 1 & $66 \cdot 1$ & $8 \cdot 5$ & $81 \cdot 0$ & $7 \cdot 4$ \\
\hline \multirow[t]{2}{*}{ Yes } & Yes & & 2 & $64 \cdot 6$ & 10.9 & 74.5 & $11 \cdot 2$ \\
\hline & Yes & Yes & 2 & $62 \cdot 5$ & $10 \cdot 0$ & $78 \cdot 6$ & $9 \cdot()$ \\
\hline Yes & & Yes & 2 & $82 \cdot 1$ & $9 \cdot 3$ & $88 \cdot 1$ & $7 \cdot 9$ \\
\hline Yes & Yes & Yes & 3 & $81 \cdot 3$ & $11 \cdot 9$ & 9()$\cdot 6$ & 8.9 \\
\hline
\end{tabular}


cytic choroidal lesions, nor are they necessarily the only important ones.

The findings of this study do not clarify the important question about whether mere recording of lesion enlargement is appropriate management of patients with suspected choroidal melanoma. The answer to this question will have to await the results of appropriately designed, comparative survival analyses of patients managed promptly after clinical diagnosis of choroidal melanoma versus those treated only after recording of lesion enlargement. Meanwhile our table showing the incidence of lesion enlargement as a function of the presence or absence of the three prognostic parameters identified by our best Cox model (Table 3) may prove useful to clinicians. Using this table a clinician can estimate the likelihood that a patient's melanocytic choroidal lesion will enlarge during up to five years of followup. If the estimated likelihood of lesion enlargement is high and the clinician elects to observe the patient rather than advise treatment, he will probably want to arrange for closely spaced follow-up evaluations. If, on the other hand, the likelihood of lesion enlargement appears to be low, the clinician may be more confident in arranging for longer intervals between follow-up examinations.

\section{References}

1 Gass JDM. Problems in the differential diagnosis of choroidal nevi and malignant melanomas. Am J Ophthalmol 1977: 83: 299-323.

2 Shields JA, Augsburger JJ, Brown GC, Stephens RF. The differential diagnosis of posterior uveal melanoma. Ophthalmology 1980: 87: 518-22.

3 Tamler E. Maumenee AE. A clinical study of choroidal nevi Arch Ophthalmol 1959; 62: 196-202.

4 Tamler E. A clincial study of choroidal nevi. A follow-up report. Arch Ophthalmol 1970: 84: 29-32.

5 Naumann GOH, Hellner K. Naumann LR. Pigmented nevi of the choroid. Clinical study of secondary changes in the overlying tissues. Ophthalmology 1971: 75: 11(1-22.

6 Mims JL. Shields JA. Follow-up studies of suspicious choroidal nevi. Ophthalmology 1978: 85: 929-43.

7 Shields JA. Current approaches to the diagnosis and management of choroidal melanomas. Surv Ophthalmol 1977: 21: 443-63.

8 Gass JDM. Observation of suspected choroidal and ciliary body melanomas for evidence of growth prior to enucleation. Ophthalmology 1987: 80: 523-8.

9 Zimmerman LE. McLean IW. Do growth and onset of symptoms of uveal melanomas indicate subclinical metatasis? Ophthalmology 1984: $91: 68.5-91$.

10 Shields JA. Diagnosis and management of intraocular tumors. St Louis: Mosby, 1983: 122-8, 140, 156-8, 172-3, 211-2.

11 Kaplan EL. Meier P. Nonparametric estimation from incomplete observations. J Am Stat Assoc 1958: 53: 437-81.

12 Cox DR. Regression models and life-tables. J R Stat Soc B 1972. 34: $187-220$.

Accepted for publication I December 1988. 